\title{
THE INTERFERENCE OF HIGHER ORDER LAGUERRE-GAUSSIAN BEAMS
}

\author{
V. Pyragaitè, V. Smilgevičius, A. Stabinis, and V. Maslinskas \\ Department of Quantum Electronics, Vilnius University, Saulètekio 9, LT-10222 Vilnius, Lithuania \\ E-mail: algirdas.stabinis@ff.vu.lt
}

Received 19 December 2006

\begin{abstract}
A vorticity of the light field created by interference of two parallel Laguerre-Gaussian vortex beams of topological charge $n>1$ is analysed. It is demonstrated that the locations of the vortices present in the composite beam depend on the separation between two beams as well as on the phase shift and topological charge. The qualitative agreement between theoretical and experimental data was obtained.
\end{abstract}

Keywords: beam interference, optical vortex

PACS: $42.25 . \mathrm{Hz}, 42.60 . J f$

\section{Introduction}

In 1974 it was shown that the scalar Helmholtz equation has a solution of the type $r^{n} \mathrm{e}^{\mathrm{i} n \theta}$ [1]. This type of solution has a singular point at radius $r=0$. The beam intensity vanishes at this point, and the azimuthal angle $\theta$ is undefined there. The integer number $n$ is the topological charge. Such a singular beam satisfies the laser model equation and was named the optical vortex [2]. The solutions of Helmholtz equation possessing Gaussian aperture are the Laguerre-Gaussian and Bessel-Gaussian beams. These beams are of practical interest because they have an orbital angular momentum [3], which can be transferred to trapped particles. A fundamental question arises about the vorticity of the light field created by superposition of individual vortex beams. In the case of superposition of two coaxial Laguerre-Gaussian beams the number of existing vortices and their net topological charge are found to depend during free-space propagation on the beam relative widths and amplitudes [4]. The interference of $m$ th-order Laguerre-Gaussian beam with a weak coaxial Gaussian beam was investigated in Ref. [5]. As a result, the Laguerre-Gaussian vortex splits into $|m|$ single-charge vortices. The interference of coaxial Bessel-Gaussian beams was studied in [6,7]. It was shown that their vortical structure is richer than that of individual beams and varies under diffraction. In the case of superposition of two noncoaxial parallel singlecharge beams $[8,9]$ the number of vortices in compos- ite beam may vary depending on the distance between beam axes as well as on the phase shift. The vorticity of such composite beam changes under diffraction of individual beams [10]. In the case of intersecting Laguerre-Gaussian beams the vortical structure of the light field depends on the intersection angle [11]. A richer vortical structure is also obtained after interference of Bessel-Gaussian and Laguerre-Gaussian beams [12].

In this paper we investigate the vortical structure of the composite beam obtained by superposition of two higher-order Laguerre-Gaussian beams and demonstrate that the superposition creates light patterns with a richer vortex content, which essentially depends on topological charges $n$ of individual beams.

\section{Theoretical}

In Cartesian coordinates the complex amplitude of Laguerre-Gaussian vortex beam of topological charge $n$ can be written as

$$
A_{n 0}(x, y)=A_{0}\left(\frac{x+\mathrm{i} y}{d}\right)^{n} \exp \left(-\frac{x^{2}+y^{2}}{d^{2}}\right),
$$

where $d$ is a beam radius at the waist. The vortex core is located at $x=0, y=0$. We analyse the superposition of two identical higher-order collinear vortex beams, the axes of which are separated by a distance $2 a$ (Fig. 1). In that case the complex amplitude of the 


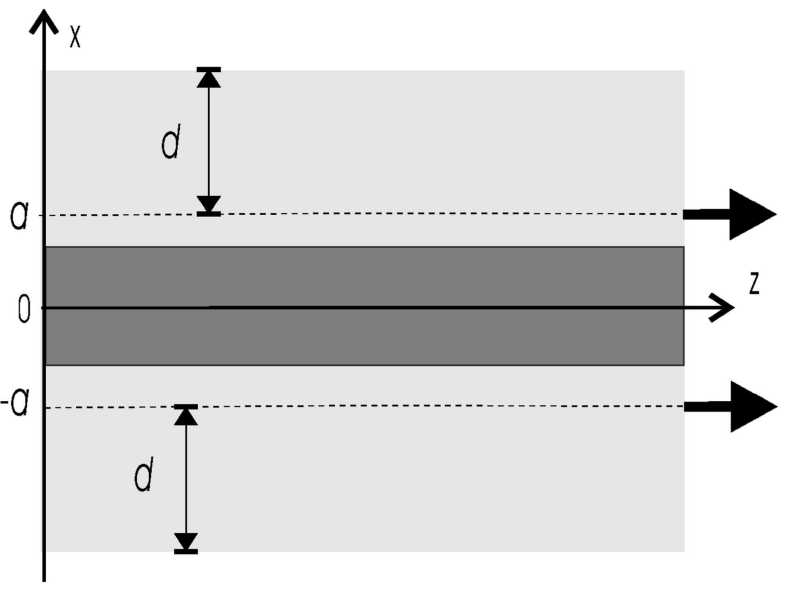

Fig. 1. Schematic depiction of the overlapping of two beams.

composite beam is

$$
A_{n}(x, y)=A_{n 0}(x-a, y)+\exp (\mathrm{i} \varphi) A_{n 0}(x+a, y),
$$

where $\varphi$ is the phase shift. It is supposed that the amplitudes and topological charges of both beams are the same. The locations of the vortex cores of the composite beam can be determined using the equations

$$
\operatorname{Re}\left[A_{n}(x, y)\right]=0, \quad \operatorname{Im}\left[A_{n}(x, y)\right]=0 .
$$

Further, we will investigate the cases $n=2$ and $n=3$ at $\varphi=0$ and analyse the influence of the phase shift $\varphi=\pi$ for $n=2$.

\section{1. $n=2, \varphi=0$}

In this case the complex amplitude of the composite beam can be written as

$$
\begin{gathered}
A_{2}(x, y)=A_{0}\left[\frac{x-a+\mathrm{i} y}{d}\right]^{2} \exp \left[-\frac{(x-a)^{2}+y^{2}}{d^{2}}\right] \\
+A_{0}\left[\frac{x+a+\mathrm{i} y}{d}\right]^{2} \exp \left[-\frac{(x+a)^{2}+y^{2}}{d^{2}}\right] .
\end{gathered}
$$

The numerically calculated distribution of the intensity $\left|A_{2}(x, y)\right|^{2}$ as a function of axial separation $a$ is depicted in Fig. 2. The locations of the vortex cores of composite beam can be found analytically by use of Eqs. (3),

$$
\begin{gathered}
{\left[(x-a)^{2}-y^{2}\right] \exp \left(\frac{2 x a}{d^{2}}\right)} \\
+\left[(x+a)^{2}-y^{2}\right] \exp \left(-\frac{2 x a}{d^{2}}\right)=0, \\
y(x-a) \exp \left(\frac{2 x a}{d^{2}}\right)+y(x+a) \exp \left(-\frac{2 x a}{d^{2}}\right)=0 .
\end{gathered}
$$

At $y \neq 0$ Eqs. (5) can be written as

$$
x=a \tanh \frac{2 x a}{d^{2}}, \quad y= \pm \sqrt{a^{2}-x^{2}} .
$$

Then for $0<a<d / \sqrt{2}$ we obtain $x=0, y= \pm a$. So, in this case in the composite beam there exist two single-charge vortices the cores of which are separated by a distance $2 a$ (see Fig. 2(b)). However, when the axial separation $a$ is equal to $d / \sqrt{2}$, the vortices change the sign of their charge, and the vortex twin is nucleated at the origin of each vortex, Fig. 2(c). As a result, there exist six vortices in the composite beam at $a>d / \sqrt{2}$, the cores of which are located at the circle given by equation $x^{2}+y^{2}=a^{2}$ (see Fig. 2(c-e)). The total charge of all vortices is always +2 . In the limiting case $a \gg d$ the solutions of Eqs. (6) are $x=0, y= \pm a$ and $x= \pm a, y=0$. These solutions correspond to two well-separated beams of charge 2 with cores at $(a, 0)$ and $(-a, 0)$. However, two vortices with the negative charge -1 are always present in the region where the field amplitude is vanishingly small, see Fig. 2(f-h) for $a / d=2$.

\section{2. $n=2, \varphi=\pi$}

The vortical structure of the composite beam may depend on the relative phase $\varphi$. In the following, we demonstrate this for a special case $\varphi=\pi$. Then the complex amplitude of the composite beam can be written as follows:

$$
\begin{gathered}
A_{2}(x, y)=A_{0}\left[\frac{x-a+\mathrm{i} y}{d}\right]^{2} \exp \left[-\frac{(x-a)^{2}+y^{2}}{d^{2}}\right] \\
-A_{0}\left[\frac{x+a+\mathrm{i} y}{d}\right]^{2} \exp \left[-\frac{(x+a)^{2}+y^{2}}{d^{2}}\right] .
\end{gathered}
$$

The numerically calculated evolution of intensity $\left|A_{2}(x, y)\right|^{2}$ is presented in Fig. 3. The locations of the vortex cores in the composite beam is determined by equations

$$
\begin{gathered}
{\left[(x-a)^{2}-y^{2}\right] \exp \left(\frac{2 x a}{d^{2}}\right)} \\
-\left[(x+a)^{2}-y^{2}\right] \exp \left(-\frac{2 x a}{d^{2}}\right)=0 \\
y(x-a) \exp \left(\frac{2 x a}{d^{2}}\right)-y(x+a) \exp \left(-\frac{2 x a}{d^{2}}\right)=0 .
\end{gathered}
$$



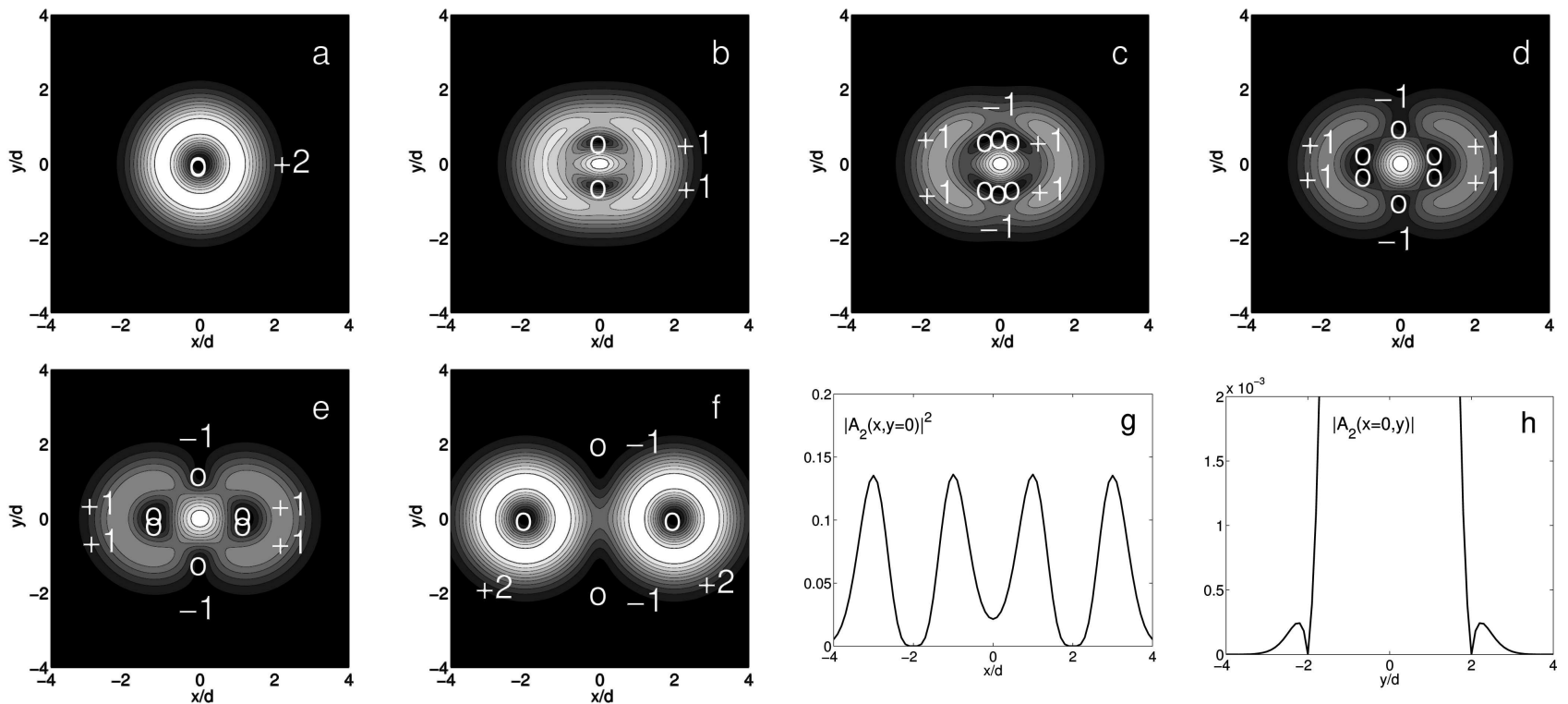

Fig. 2. Intensity distribution of the composite beam for $n=2, \varphi=0$, and at $a / d$ : (a) 0 , (b) 0.6 , (c) 0.74 , (d) 1 , (e) 1.2 , (f) 2 . The circles mark the locations of the vortex cores. $(\mathrm{g})\left|A_{2}(x, y)\right|^{2}$ profile at $y=0$ and $(\mathrm{h})\left|A_{2}(x, y)\right|$ profile at $x=0$, when $a / d=2$.
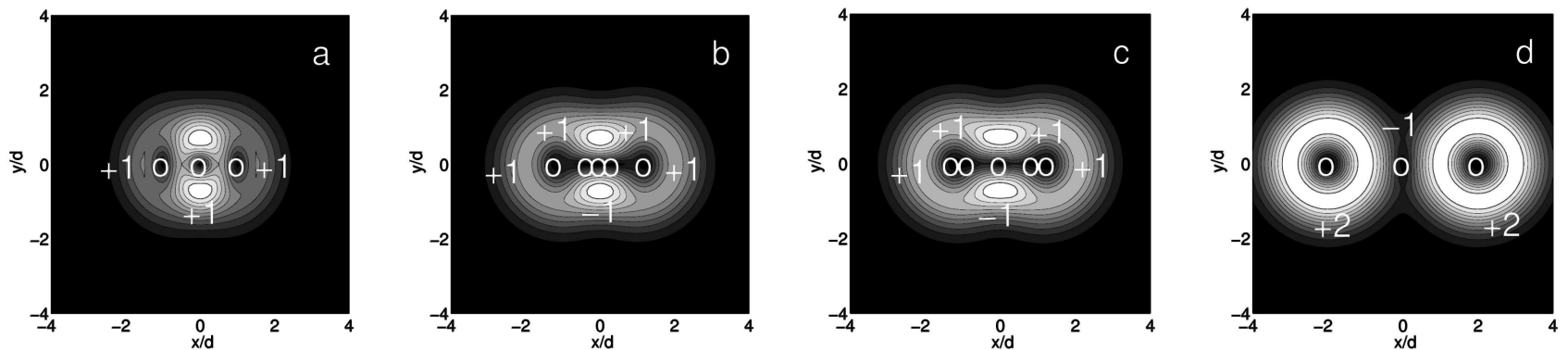

Fig. 3. Intensity distribution of the composite beam for $n=2, \varphi=\pi$, and at $a / d$ : (a) 0.3 , (b) 1.02 , (c) 1.14 , (d) 2 . The circles mark the locations of the vortex cores.

An analysis of Eqs. (8) shows that the solutions exist only for $y=0$. In this case the first equation of Eqs. (8) can be written as

$$
\left(x^{2}+a^{2}\right) \tanh \frac{2 a x}{d^{2}}=2 a x .
$$

Obviously, a trivial solution of Eq. (9) at $a \neq 0$ is $x=$ 0 . The vortices are also located at $x \neq 0$. For example, at $a / d \ll 1$ we find $x= \pm d$. So, three vortices are present in the composite beam at $y=0$ (see Fig. 3(a)). Next, for small values $x$ and $y$ from Eq. (7) we obtain

$$
A_{2}(x, y) \propto\left(1-\frac{a^{2}}{d^{2}}\right) x+\mathrm{i} y .
$$

At the critical value $a=d$ the central vortex changes its topological charge from +1 to -1 and two additional vortices of charge +1 appear (Fig. 3(b)). If the separation between two individual beams is further increased, these vortices collide with the vortices at $x \neq 0$ and form two double-charge vortices, Fig. 3(c, d). We note, that one negative-charge $(-1)$ vortex is located at infinity $(x=0, y=-\infty)$ and for this reason the total charge of the composite beam is +2 .

\section{3. $n=3, \varphi=0$}

In this case Eq. (2) yields

$$
\begin{array}{r}
A_{3}(x, y)=A_{0}\left[\frac{x-a+\mathrm{i} y}{d}\right]^{3} \exp \left[-\frac{(x-a)^{2}+y^{2}}{d^{2}}\right] \\
+A_{0}\left[\frac{x+a+\mathrm{i} y}{d}\right]^{3} \exp \left[-\frac{(x+a)^{2}+y^{2}}{d^{2}}\right] .
\end{array}
$$

The numerically calculated evolution of the intensity $\left|A_{3}(x, y)\right|^{2}$ on the axial separation $a$ is depicted in Fig. 4. The locations of the vortex cores in the com- 

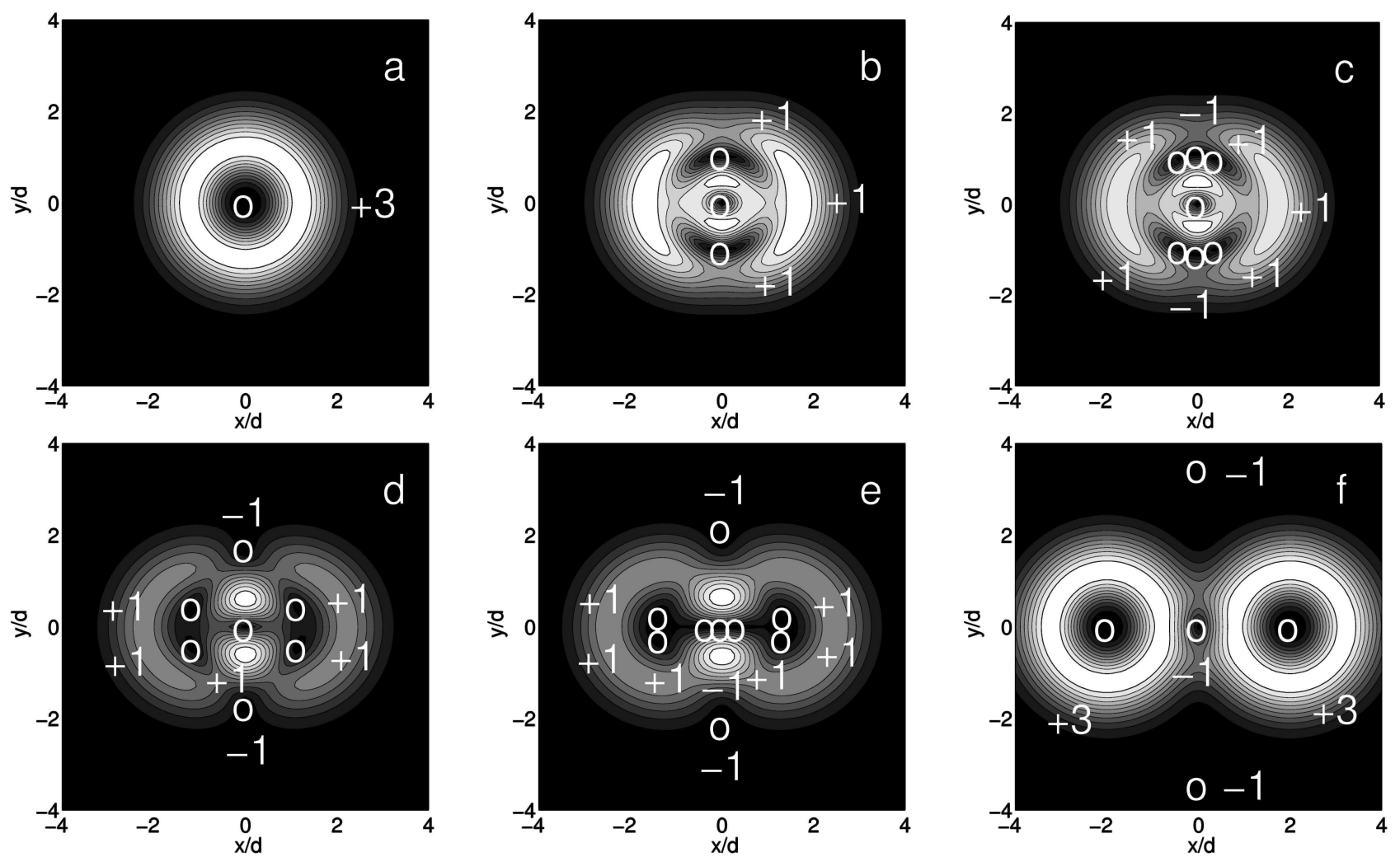

Fig. 4. Intensity distribution of the composite beam for $n=3, \varphi=0$, and at $a / d$ : (a) 0 , (b) 0.6 , (c) 0.64 , (d) 1 , (e) 1.24 , (f) 2 . The circles mark the locations of the vortex cores.

posite beam can be determined by use of Eqs. (3). We obtain

$$
\begin{gathered}
{\left[(x-a)^{3}-3 y^{2}(x-a)\right] \exp \left(\frac{2 x a}{d^{2}}\right)} \\
+\left[(x+a)^{3}-3 y^{2}(x+a)\right] \exp \left(-\frac{2 x a}{d^{2}}\right)=0 \\
\quad\left[3 y(x-a)^{2}-y^{3}\right] \exp \left(\frac{2 x a}{d^{2}}\right) \\
+\left[3 y(x+a)^{2}-y^{3}\right] \exp \left(-\frac{2 x a}{d^{2}}\right)=0 .
\end{gathered}
$$

We note that for $a>0$ at $x=0$ the solutions of Eqs. (12) are $y=0, \pm \sqrt{3} a$ (Fig. 4(b)). In the vicinity of the points $(0, \pm \sqrt{3} a)$ from Eq. (11) for small values of $\Delta x, \Delta y$ it follows that

$$
\begin{aligned}
A_{3}(\Delta x, y & = \pm \sqrt{3} a+\Delta y) \\
& \propto\left(3 d^{2}-8 a^{2}\right) \Delta x+\mathrm{i} 3 d^{2} \Delta y .
\end{aligned}
$$

So, there is a critical value $a=a_{1}=\sqrt{3 / 8} d \approx 0.61 d$ at which the topological charge of the vortices at $(0$, $\pm \sqrt{3} a$ ) is changed from +1 to -1 , and the vortex twin is nucleated at the origin at each vortex, see Fig. 4(c). As a result, at $a>a_{1}$ four positively charged $(+1)$ vortices are present in the composite beam. Next, in the vicinity of the point $(0,0)$ the Eq. (11) can be written as

$$
A_{3}(x, y) \propto\left(3 d^{2}-2 a^{2}\right) x+3 \mathrm{i} d^{2} y .
$$

So, at $a=a_{2}=\sqrt{3 / 2} d \approx 1.22 d$ the central vortex changes its sign from +1 to -1 , and simultaneously two new vortices of charge +1 appear, Fig. 4(e). If the separation between two individual beams is further increased, the positively charged vortices approach each other and in the limit $a / d \gg 1$ merge into two vortices of charge +3 . In this case the solutions of Eqs. (12) at $y=0$ are $x \rightarrow \pm a$. However, three vortices with negative charge -1 are always present in the region where the field amplitude is vanishingly small, as shown in Fig. 4(f) for $a / d=2$. The total charge of all vortices is always equal to 3 .

\section{Experimental}

An experimental set-up is presented in Fig. 5. In experiments the linearly polarized radiation of second 


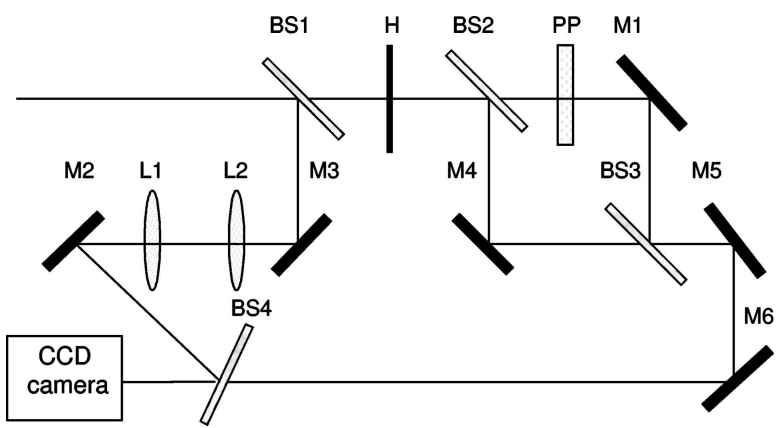

Fig. 5. Experimental set-up. L1 and L2 are lenses, BS1-BS4 are beam splitters, M1-M6 are mirrors, PP is a parallel plate, $\mathrm{H}$ is a hologram.

harmonic (SH) of a microchip Nd:LSB (lanthanum scandium borate) laser (STA-1) was used $(\lambda=531 \mathrm{~nm})$. The $\mathrm{SH}$ radiation was filtered by spatial filter and collimated into $d=1.2 \mathrm{~mm}$ diameter beam. The obtained beam was divided into two beams by a beam splitter BS1. One of them was directed to a computergenerated hologram $\mathrm{H}$, and 2nd or 3rd higher-order Laguerre-Gaussian beams were produced. The second beam was used as a reference beam for analysis of topological charges by interference with the composite beam, which was formed by use of beam splitters (BS2, BS3) and mirrors (M1, M4). The phases of the vortex beams were adjusted by a parallel glass plate (PP). The interference patterns were registered by a CCD camera and are presented for $n=3$ in Fig. 6. The obtained patterns can be compared with the numerically calculated patterns shown in Fig. 4. Obviously, the qualitative agreement between theoretical and experimental data is obtained.

\section{Conclusions}

The interference of two parallel higher-order Laguerre-Gaussian beams was investigated. It was revealed that the number of vortices as well as their loca- tions in the composite beam depend on the separation between beam axes, relative phase shift of the beams, and topological charge. Very similar scenarios of the evolution of vortical structure on beam separation in the case of second- and third-order Laguerre-Gaussian beams were observed. In the case of small separation of two individual beams in one direction all vortices are found to be located on the straight line in the perpendicular direction. The vortices change their sign at some critical value of the beam separation, which is different for different vortices and depends on the vortex distance from the centre $(x=y=0)$. Simultaneously, two additional vortices are nucleated at the origin of each vortex. In the case of large separation, two wellseparated beams of charge $n$ are obtained. However, the total charge of a light field is $n$, because $n$ singlecharge $(-1)$ vortices are present in the region where the field amplitude is vanishingly small.

The qualitative agreement between theory and experiment was obtained.

\section{References}

[1] J.F. Nye and M.V. Berry, Dislocations in wave trains, Proc. R. Soc. London, Ser. A 336, 165-190 (1974).

[2] P. Coullet, L. Gil, and F. Rocca, Optical vortices, Opt. Commun. 73, 403-407 (1989).

[3] L. Allen, M.W. Beijersbergen, R.J.C. Spreeuw, and J.P. Woerdman, Orbital angular momentum of light and the transformation of Laguerre-Gaussian laser modes, Phys. Rev. A 45, 8185-8189 (1992).

[4] M.S. Soskin, V.N. Gorshkov, M.V. Vasnetsov, J.T. Malos, and N.R. Heckenberg, Topological charge and angular momentum of light beams carrying optical vortices, Phys. Rev. A 56, 4064-4075 (1997).

[5] I.V. Basistiy, V.Yu. Bazhenov, M.S. Soskin, and M.V. Vasnetsov, Optics of light with screw dislocations, Opt. Commun. 103, 422-428 (1993).

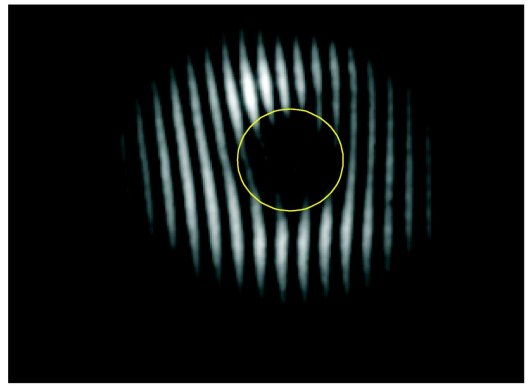

(a)

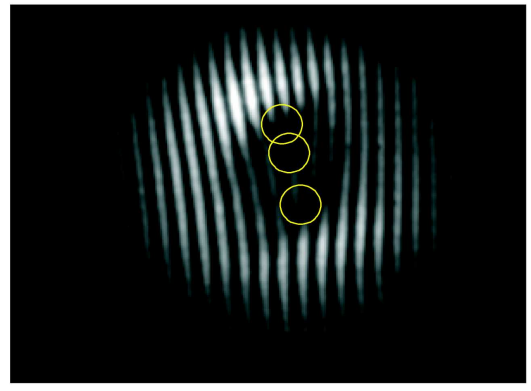

(b)

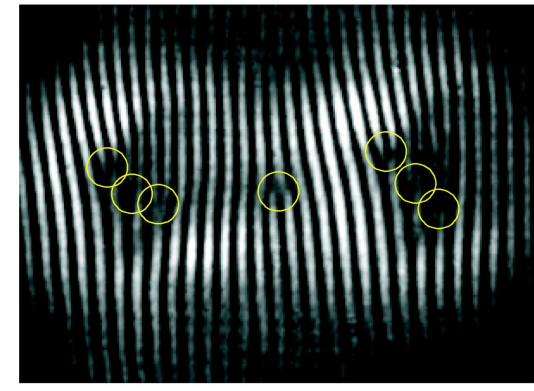

(c)

Fig. 6. Interference patterns for $n=3, \varphi=0$, and at $a / d$ : (a) 0 , (b) 0.3 , (c) 1.44 . The circles mark the locations of the vortex cores. 
[6] S. Orlov, K. Regelskis, V. Smilgevicius, and A. Stabinis, Propagation of Bessel beams carrying optical vortices, Opt. Commun. 209, 155-165 (2002).

[7] S. Orlov and A. Stabinis, Propagation of superpositions of coaxial optical Bessel beams carrying vortices, J. Opt. A: Pure Appl. Opt. 6, S259-S262 (2004).

[8] G. Molina-Terriza, J. Recolons, and L. Torner, The curious arithmetic of optical vortices, Opt. Lett. 25, 1135-1137 (2000).

[9] I.D. Maleev and G.A. Swartzlander, Composite optical vortices, J. Opt. Soc. Am. B 20, 1169-1176 (2003).
[10] V. Pyragaite and A. Stabinis, Free-space propagation of overlapping light vortex beams, Opt. Commun. 213, 187-191 (2002).

[11] V. Pyragaite and A. Stabinis, Interference of intersecting singular beams, Opt. Commun. 220, 247-255 (2003).

[12] S. Orlov and A. Stabinis, Free-space propagation of light field created by Bessel-Gauss and LaguerreGauss singular beams, Opt. Commun. 226, 97-105 (2003).

\title{
AUKŠTESNĖS EILĖS LAGERO IR GAUSO PLUOŠTU INTERFERENCIJA
}

\author{
V. Pyragaitè, V. Smilgevičius, A. Stabinis, V. Maslinskas \\ Vilniaus universitetas, Vilnius, Lietuva
}

\section{Santrauka}

Nagrinejjama dviejų lygiagrečiu Lagero ir Gauso (LaguerreGauss) sūkurinių topologinio krūvio $n>1$ pluoštų interferencijos lauko sūkurinė sandara. Parodyta, kad sudètiniame pluošte esančių sūkurių padètys priklauso tiek nuo atstumo tarp pluoštų, tiek ir nuo fazių skirtumo bei topologinio krūvio. Gautas kokybinis teorinių ir eksperimentinių duomenų sutapimas. 